\title{
COMPARISON OF PATIENT CONTROLLED EPIDURAL ANALGESIA WITH CONTINUOUS EPIDURAL INFUSION FOR LABOUR ANALGESIA
}

\author{
Sumaiah Tahseen ${ }^{1}$, Kassim Mohammed ${ }^{2}$, Sahajananda $H^{3}$, Praveen B. Halagunaki ${ }^{4}$
}

${ }_{1}^{1}$ Post Graduate Student, Department of Anaesthesia, Rajarajeswari Medical College and Hospital, Bangalore. 2Post Graduate Student, Department of Anaesthesia, Rajarajeswari Medical College and Hospital, Bangalore. ${ }^{3}$ Professor and HOD, Department of Anaesthesia, Rajarajeswari Medical College and Hospital, Bangalore. ${ }_{4}^{4}$ Post Graduate Student, Department of Anaesthesia, Rajarajeswari Medical College and Hospital, Bangalore.

\begin{abstract}
We conducted a study to compare the efficacy and safety of Patient Controlled Epidural Analgesia (PCEA) with that of Continuous Infusion of Epidural Analgesia (CIEA) for maintenance of labour analgesia and evaluated the quality of analgesia and obstetric and safety outcomes.

\section{METHODS}

The study was a hospital-based prospective, randomised control trial on 80 parturients who had a normal antenatal period. Each parturient received 500-1000 mL lactated ringer solution Intravenously (IV) prior to initiating epidural blockade. Epidural catheter placement was performed in a standard manner and all patients received an initial dose of 8-10 mL bupivacaine 0.25\%. Parturients self-administered $0.125 \%$ bupivacaine with fentanyl $2.5 \mu \mathrm{g} / \mathrm{mL}$ using PCA pumps programmed as follows: $4 \mathrm{~mL}$ bolus with a $20 \mathrm{mins}$

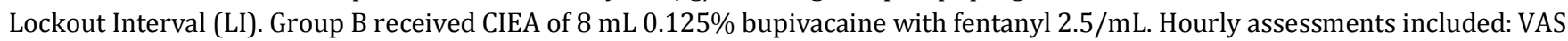
scores for pain and satisfaction, sensory and motor block, analgesic supplements, bupivacaine and fentanyl consumption.
\end{abstract}

\section{RESULTS}

Data from 80 patients showed no differences among groups in pain relief. Maternal satisfaction was greater in PCEA group. Anaesthetic interventions by way of supplemental doses of Bupivacaine and Fentanyl in the PCEA group were minimal ( 4 and 2 vs 25 and $12 \mathrm{P}<0.001)$ compared to CEI group. PCEA group received less local anaesthetic $(5.2$ vs $9.4 \mathrm{p}<0.001)$ and few patients in PCEA group had motor weakness compared to CEI group ( 6 vs $17 \mathrm{p}<0.05$ ). Both methods were safe for mother and newborn.

\section{CONCLUSION}

Patients who received PCEA required less anaesthetic interventions, required lower doses of local anaesthetic, fentanyl and have less motor weakness than those who received CEI.

\section{KEYWORDS}

Patient Controlled Analgesia, Fentanyl, Epidural Analgesia.

HOW TO CITE THIS ARTICLE: Tahseen S, Mohammed K, Sahajananda H, et al. Comparison of patient controlled epidural analgesia with continuous epidural infusion for labour analgesia. J. Evolution Med. Dent. Sci. 2016;5(56):3862-3867, DOI: $10.14260 /$ jemds/2016/884

\section{INTRODUCTION}

Pain is the most stressful moment during labour. A number of techniques have been evolved to maintain the level of analgesia throughout. ${ }^{1}$ Epidural analgesia has become very popular and widely used in the clinical practice. Historically, intermittent bolus dosing of local anaesthetic by the clinician, anaesthetist, nurse or technician was used. However, this technique had a number of drawbacks including inconsistent analgesia, inherent toxicity and concerns about sterility each time the clinician opened the system to administer a bolus. ${ }^{1}$ Continuous epidural infusion of local anaesthetics was introduced into common clinical practice in 1980 and has circumvented a number of difficulties. Although, many combinations of infusion rates and various concentrations of local anaesthetics and additives have been investigated, but problems still persist. ${ }^{2}$ Patient-controlled epidural analgesia,

Financial or Other, Competing Interest: None.

Submission 31-03-2016, Peer Review 02-07-2016,

Acceptance 07-07-2016, Published 14-07-2016.

Corresponding Author:

Sahajananda $H$,

\#413, Ragam, $7^{\text {th }}$ Main,

Vijaya Bank Colony,

Bannerghatta Road,

Bangalore-76.

E-mail: sahaj_anands@hotmail.com

DOI: $10.14260 /$ jemds/2016/884 which was described in 1988 by Gambling, allows the patient to control the dose of epidural medication as labour and pain patterns changed, individualization of drug dosage with maximum therapeutic effects and lesser side-effects. ${ }^{3}$ Dosing delays are avoided. Self-administration of small increments of dilute local anaesthetic is more likely to avoid the adverse consequences of large bolus administration. However, the facilities and equipment like PCA pump needed for PCEA may be more expensive than pump required for CEI. More time is required to educate both the patient and staff about the appropriate use of the medication and equipment. ${ }^{3}$ We conducted a study to compare the efficacy and safety of PCEA with continuous epidural infusion for maintenance of labour analgesia.

\section{METHODS}

After Institutional Ethical Review Board approval and informed consent nulliparous, ASA I parturients with uncomplicated, singleton pregnancies, 18 yrs. or more, in established labour and requesting epidural analgesia were recruited. Women with severe medical or obstetric complications, multiple gestation, contraindication to epidural analgesia, local anaesthetic or fentanyl allergy were excluded from the study. Each parturient received 500-1000 mL ringer lactate IV infusion prior to initiating epidural blockade. Epidural catheter placement was performed with a loss of 
resistance to saline in a standardized manner and all patients were given an initial dose of $8-10 \mathrm{~mL}$ bupivacaine $0.25 \%$.

Patients were instructed in the use of the Bard PCA| pump and then randomly assigned to one of two groups (A= PCEA and $B=C I E A)$. Study parturients were randomised as per the computer based randomised table and were placed in either of the two Group A PCEA and Group B CIEA. Each group received bupivacaine $0.125 \%$ with epinephrine and fentanyl $2.5 \mu \mathrm{g} / \mathrm{mL}$ through the pump. An infusion rate of $8 \mathrm{~mL} / \mathrm{hr}$ was selected for CIEA, as it is an infusion rate commonly used in our institution. The PCA demand button was used, but made inactive for these patients. The study protocol allowed for changing the infusion rate in order to maintain the sensory level between T10 and T6. The PCA pump was programmed by a second investigator after referring to a previously constructed randomization table. All patients including those in Group A were told to press the demand button when pain returned and to expect some relief within five to ten minutes. Supplemental epidural analgesia was provided if patients failed to get adequate pain relief after 30 mins despite making numerous demands at that time. Additional boluses of $0.125 \%$ bupivacaine with adrenaline were used to overcome inadequate sensory levels and fentanyl (25-50 $\mu \mathrm{g}$ ) was given for back pain and/or perineal discomfort, in the presence of bilateral T10 sensory levels. If these supplements were inadequate, a full "top-up" dose of $10 \mathrm{~mL}$ bupivacaine $0.125 \%$ with epinephrine was injected. If this was unsuccessful, the patient was removed from the study.

The study started when the PCEA pump was attached to the epidural catheter and ended with the onset of the second stage. Every hour an investigator collected the following data: $10 \mathrm{~cm}$ VAS scores for pain, satisfaction, maximum pain/minimum satisfaction in the preceding hour, sensory levels (Using cold cotton swab), motor block, maternal BP and Apgar sore at 0 and 5 minutes. Other data included hourly bupivacaine and fentanyl requirements including all supplements but excluding the initial dose of bupivacaine, the ratio of successful to total PCA demands, outcome of labour, one and five mins Apgar scores and a global evaluation of analgesia and satisfaction by patient following completion of the study.

Statistical analysis was performed using chi-square analysis, ANOVA groups at each time and repeated measures analysis of variance to consider all time points in a single analysis. Demographic data were compared using one-way analysis of variance. Total analgesic supplements between groups were compared using chi-square tests. A P-value $<0.05$ was considered to be statistically significant.

\section{Sample Size}

The difference between two means of visual analogue score is 2 and mean standard deviation is $2 .{ }^{4}$ Power of study was conducted with confidence limit of $80 \%$ with calculated sample size by allowing an $\alpha$ of 0.001 and $\beta$ of 0.2 per group is 37. As all the distributions will merge into normal distribution, sample size, i.e. 40 is enough because inference that can be drawn based on 40 observations will more or less remain the same in spite of any increase in the sample size. ${ }^{5}$ Hence, a total of 80 subjects were included in our study and divided into two groups each containing 40 subjects.

\section{RESULTS AND OBSERVATION}

The study done was a hospital-based prospective, randomised control clinical study conducted on consenting obstetric parturient who had a normal antenatal period, admitted as inpatients.

\section{Age Distribution}

In our study, the mean age group in our study was 22 years in the CIEA group and 21.8 years in the PCEA group and not significant (Table 1, Graph 1).

\section{Parity Index}

In our study, all the patients recruited were primiparous (Table 2, Graph 2).

\section{ASA Grade}

In our study, on evaluation of the ASA categories, parturients belonged to the ASA category 1 and statistically not significant.

\section{Sensory Levels}

Sensory levels were similar among groups, except at the third and fourth hourly intervals where higher levels were seen in both the Groups and statistically not significant (Graph 3).

\section{Motor Block}

Motor weakness was minimal in PCEA group compared to CIEA; 34 patients in the PCEA group did not have motor weakness compared to 23 in the CIEA group ( $<<0.05)$ (Table 3, Graph 4).

\section{Bupivacaine and Fentanyl Consumption}

In Group PCEA, bupivacaine consumption was 5.2 compared to 9.4 in the CIEA group ( $\mathrm{p}<0.001)$ (Graph 5).

In Group PCEA, Fentanyl consumption was 12.2 compared to 19.6 in the CIEA group $(\mathrm{p}<0.05)$ (Graph 6).

\section{Epidural Supplements}

4 patients in the PCEA group received bupivacaine epidural supplements for inadequate analgesia compared to CIEA group, which received 25 supplements. (Table 7, Graph 10).

2 patients in the PCEA group received Fentanyl epidural supplements for inadequate analgesia compared to CIEA group, which received 12 supplements $(p<0.001)$ (Table 7 , Graph 10).

\section{Apgar Score}

In our study on evaluation of the APGAR SCORE at 0 , it was 6.95 in the CIEA group and in PECA group it was 6.73. This was statistically not significant with a p value of 0.3231 (Graph 7). In our study, on evaluation of the APGAR SCORE at 5 minutes it was 8.68 in the CIEA group and in PCEA group it was 8.4. This was statistically not significant with a $\mathrm{p}$ value of 0.2406 (Graph 8).

\section{Next Time Preference}

In our study on evaluation of the satisfaction index of the use of labour analgesia in labour, in the CIEA group 70\% preferred to use it next time as compared to the PCEA group 95\% preferred to use it next time. This was statistically significant with a $p$ value of $<0.001$ (Table 3 , Graph 9).

No maternal hypotension or foetal bradycardia was observed in both the groups. No neonate required resuscitation at birth.

We could not measure the fentanyl concentration in maternal or umbilical samples.

\begin{tabular}{|c|c|c|}
\hline Age & PCEA & CIEA \\
\hline Mean & 21.8 & 22 \\
\hline P & \multicolumn{3}{|c|}{0.8431} \\
\hline \multicolumn{3}{|c|}{ Table 1: Age Distribution } \\
\hline
\end{tabular}




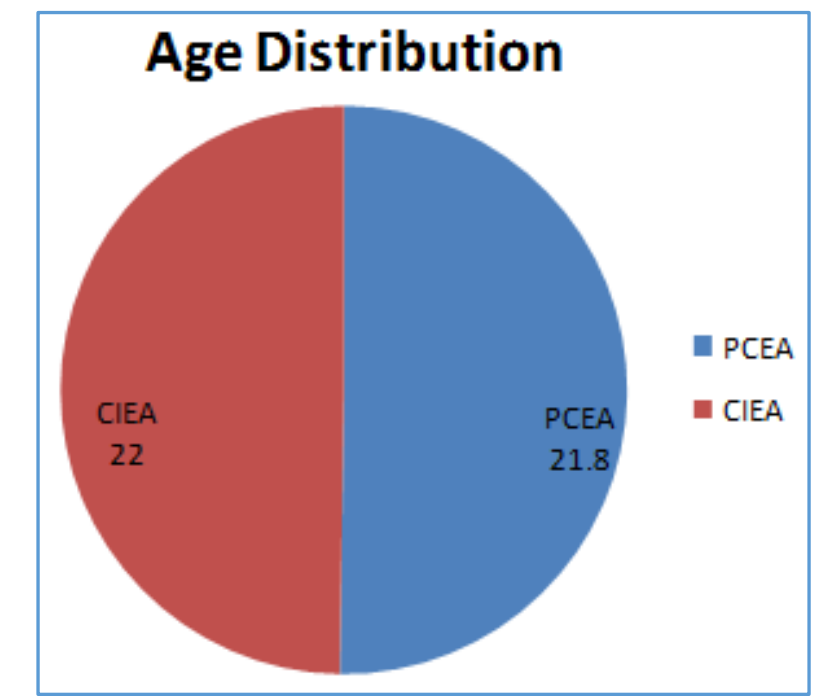

NS

\section{Graph 1: Age Distribution}

\begin{tabular}{|c|c|c|}
\hline Parity Index & CIEA & PCEA \\
\hline Primipara & 40 & 40 \\
\hline \multicolumn{2}{|c|}{ Table 2: Parity Index } \\
\hline
\end{tabular}

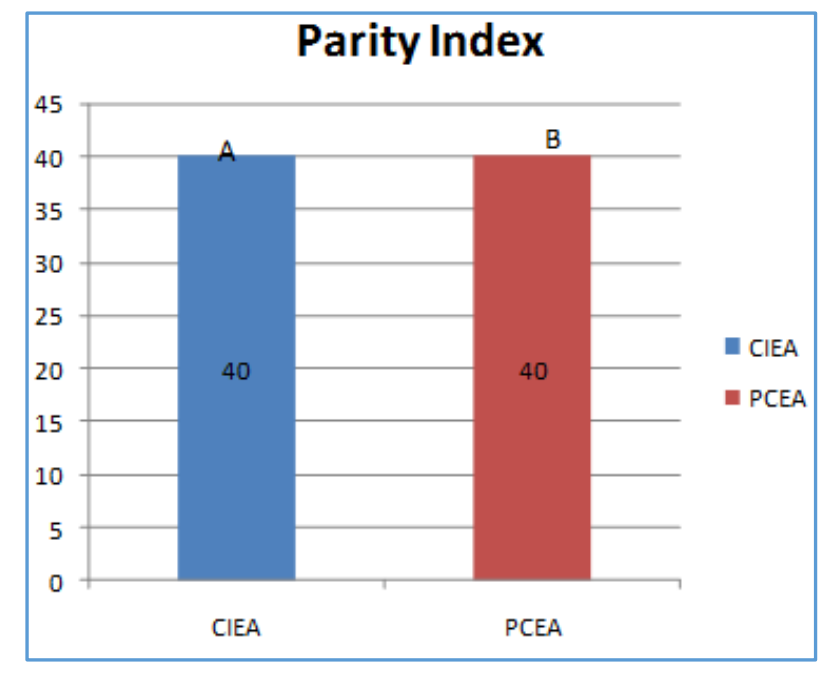

NS

Graph 2

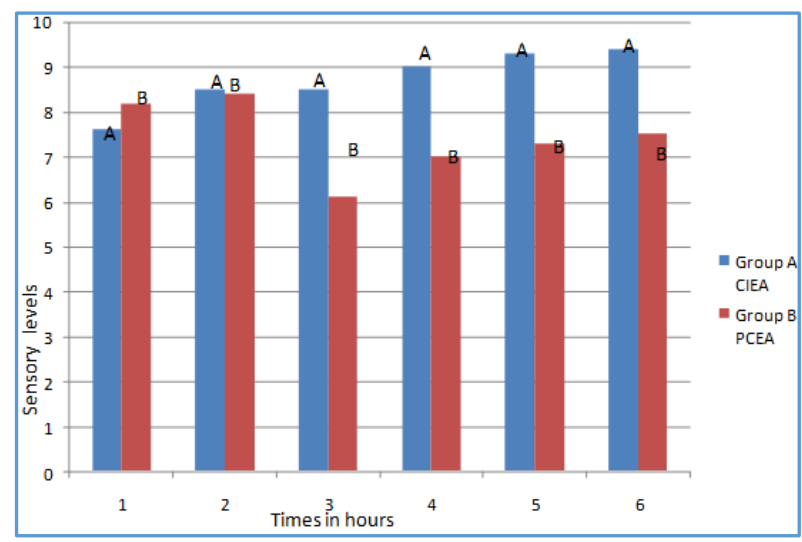

Thoracic Sensory Level

Graph 3

\begin{tabular}{|c|c|c|}
\hline Groups & A & B \\
\hline Number of patients & $34 / 40$ & $23 / 40$ \\
\hline \multicolumn{2}{|c|}{ Table 3: No. of Patients Without Motor Weakness } \\
\hline
\end{tabular}

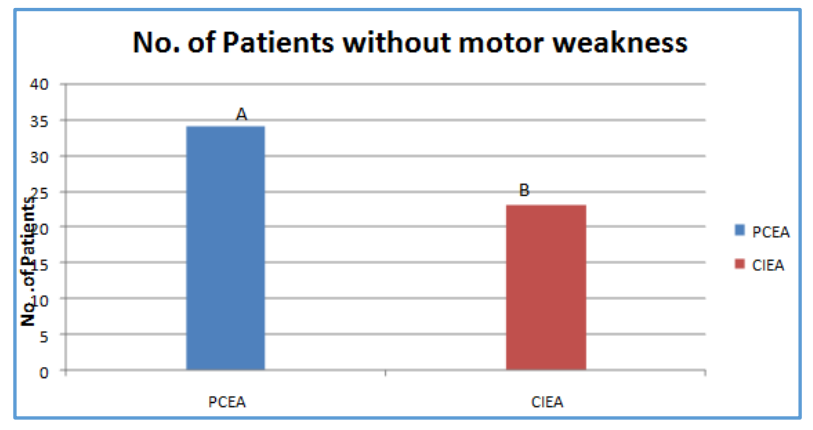

$\mathrm{P}<0.05$

Graph 4

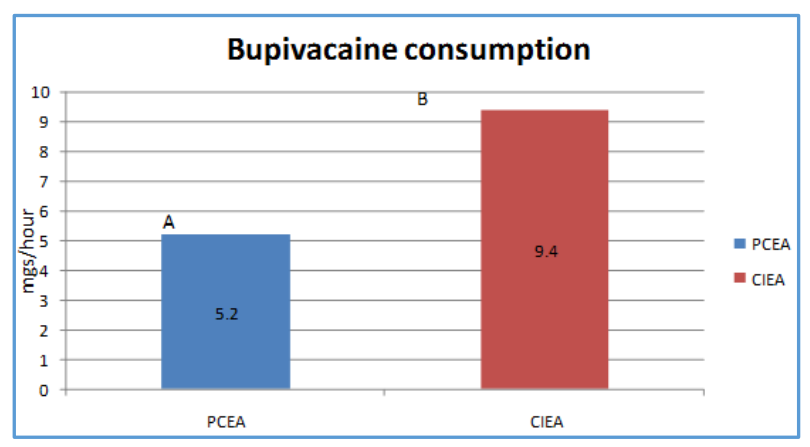

$* \mathrm{P}<0.001$

Graph 5

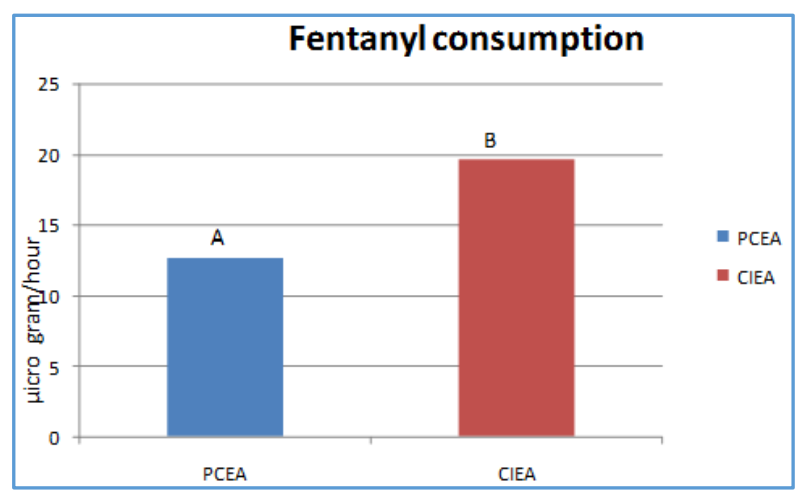

$* \mathrm{P}<0.05$

Graph 6

\begin{tabular}{|c|c|l|c|}
\hline Group & PCEA & & CIEA \\
\hline Mean & 6.95 & & 6.73 \\
\hline SD & 0.93 & & 1.09 \\
\hline SEM & 0.15 & & 0.17 \\
\hline N & 40 & & 40 \\
\hline \multicolumn{3}{|c|}{ Table 4: Apgar Score at 0 } \\
\hline
\end{tabular}

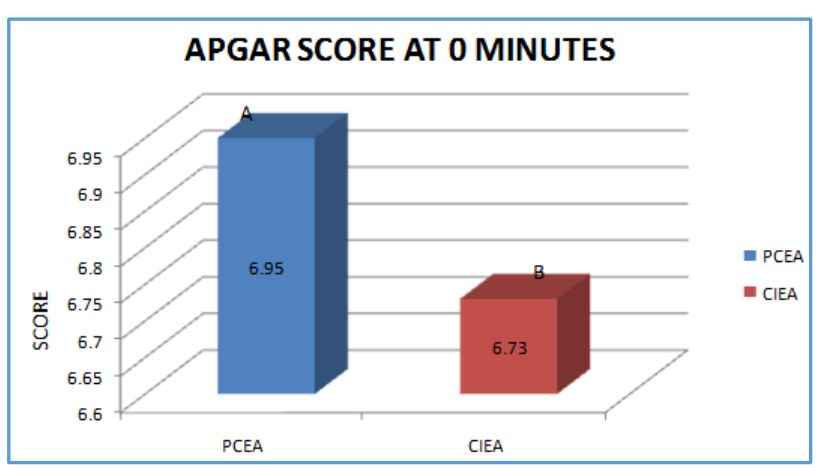

$\mathrm{P}<0.321$

Graph 7 


\begin{tabular}{|c|c|c|}
\hline Group & PCEA & CIEA \\
\hline Mean & 8.68 & 8.4 \\
\hline \multicolumn{2}{|c|}{ Table 5: Apgar Score at 5 Minutes } \\
\hline
\end{tabular}

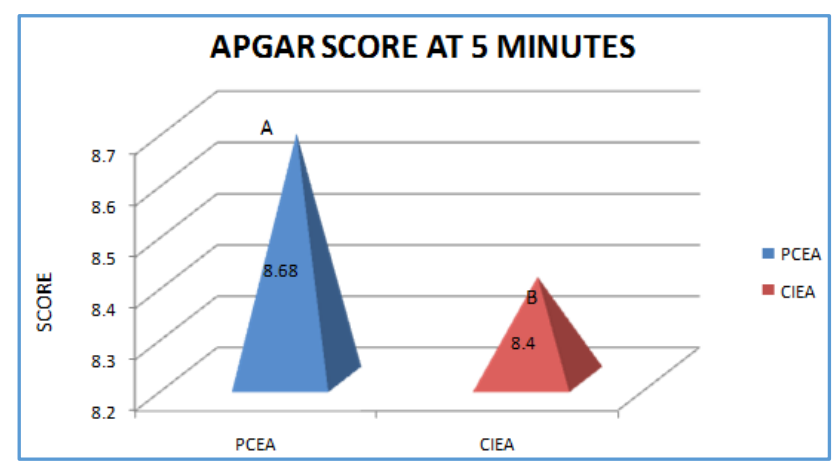

$\mathrm{P}<0.2406$

Graph 8

\begin{tabular}{|c|c|c|}
\hline Next Time Preference & Yes & No \\
\hline CIEA & $28(70 \%)$ & $12(30 \%)$ \\
\hline PCEA & $38(95 \%)$ & $2(5 \%)$ \\
\hline \multicolumn{2}{|c|}{ Table 6: Next Time Preference } \\
\hline
\end{tabular}
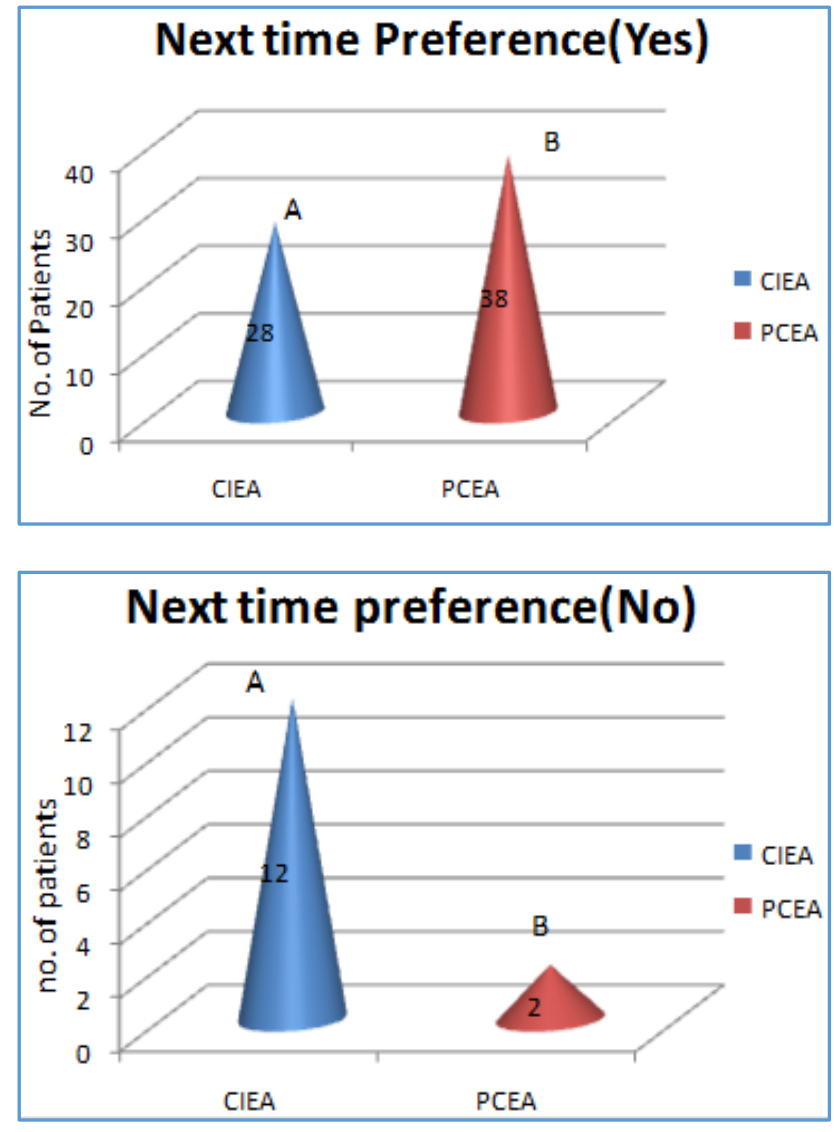

$\mathrm{P}<0.001$

Graph 9

\begin{tabular}{|c|c|c|}
\hline Supplement & A & B \\
\hline Bupivacaine & 4 & 25 \\
\hline Fentanyl & 2 & 12 \\
\hline Table 7: No. of Supplemental Doses Given for Group \\
\hline
\end{tabular}
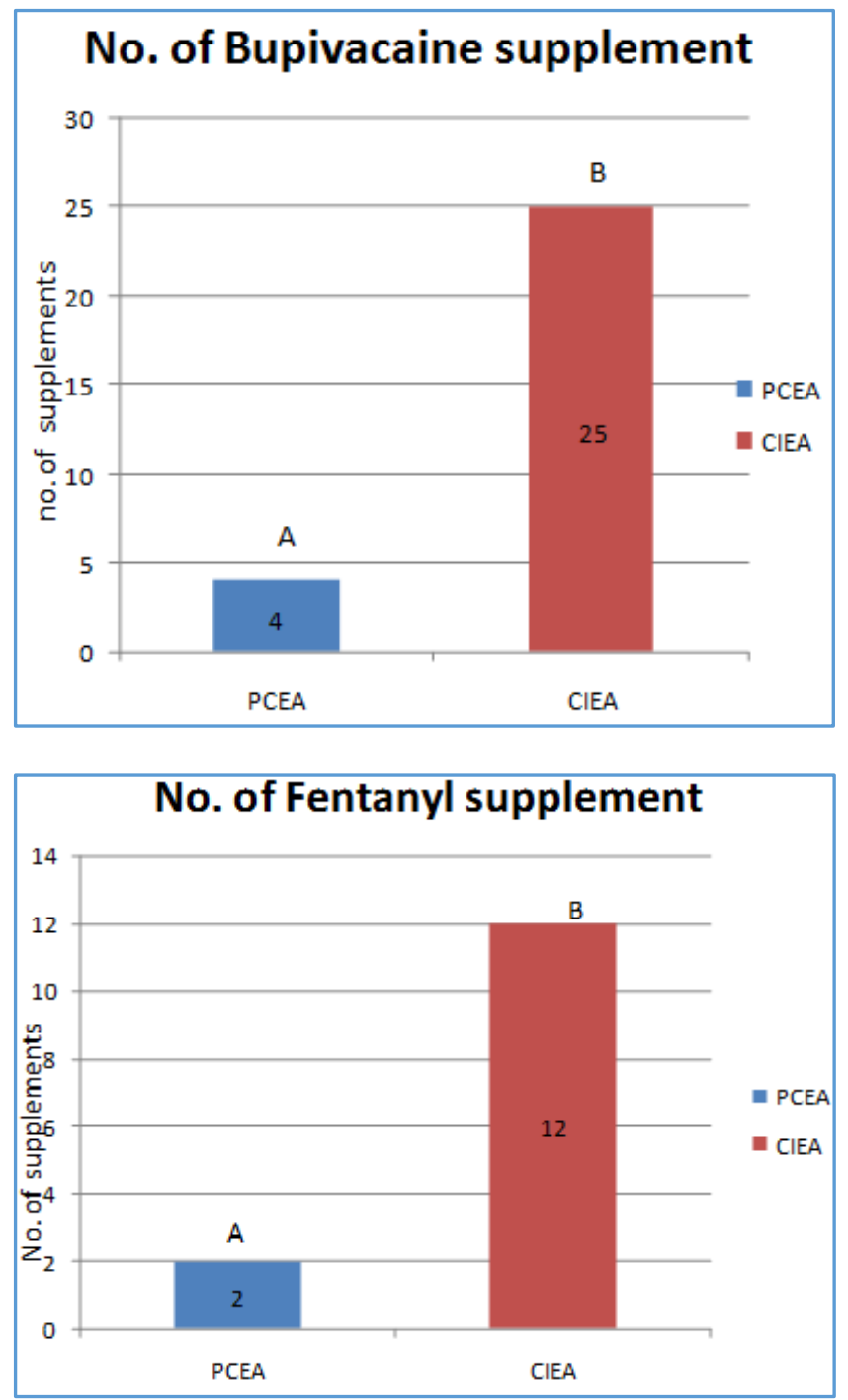

$\mathrm{P}<0.001$

Graph 10

\section{DISCUSSION}

The delivery of the infant into the arms of a conscious and pain-free mother is one of the most exciting and rewarding moments in medicine. ${ }^{6}$ In the absence of a medical contraindication, maternal request is a sufficient medical indication for pain relief during labour. With the increasing awareness among the female population in the developing world, most patients are aware of the term labour analgesia providing a memorable delivery is every obstetrician's wish and the parturients dream. In the present day obstetrics, the anaesthetist plays a very vital role along with the obstetrician during the time of child birth, especially when there is a demand for the pain free labour. Keeping this in mind, we conducted a study to compare the Patient Controlled-Epidural Analgesia (PCEA) with Continuous Infusion of Epidural Analgesia Technique (CIEA) during labour.

The need for unscheduled clinician interventions is an important outcome for a number of reasons, apart from simple convenience. Additional drug doses require opening the 'closed system' and may increase the risk of infection. Furthermore, it is usual for the clinician to use an increased drug dose compared with that available to the patient. If this dose is misplaced intravascularly or intrathecally, toxicity might result. Finally, because of the increased drug dose, motor block of the lower extremities may be more evident. 
In our study, percentage of patients receiving either a bupivacaine and fentanyl supplement ranged from $10 \%$ in Group PCEA to a $62.2 \%$ in Group CIEA (See Table No). Overall, $7.5 \%$ of all parturients receiving PCEA required additional supplementary boluses, which is comparable with a report by Paech. ${ }^{7}$ in a previous study. We are in agreement with Boutros et al ${ }^{8}$ study with bupivacaine and sufentanil, which they found it as a valuable technique and a good alternative to the CIEA method. PCEA allows a decrease in local anaesthetic consumption without impairing the quality of anaesthesia. Tan and Colleagues ${ }^{9}$ found a significantly higher number of top-ups in the control group than in the PCEA group (120 top-ups in 84 women compared with 49 in 75 women, respectively). Curry and Colleagues ${ }^{10}$ reported 27 top-ups in 30 patients who received control compared with only two top-ups in 30 patients who received PCEA $(P<0.001)$. Sia and Colleagues ${ }^{11}$ reported six top-ups in 20 patients in the control group compared with three top-ups in 20 patients in the PCEA group during the first stage of labour $(\mathrm{P}=0.4)$.

In our study, on evaluation of the APGAR SCORE at 0 and 5 minutes between the CIEA group and PCEA group, there was no statistically significant difference suggesting that there was no effect of the anaesthesia on the foetus and that labour analgesia may be used without any side effects to the foetus. Both the techniques are safe with good neonatal outcome. No adverse sequel were seen, such as maternal hypotension or fetal bradycardia.

Differences in the way PCA is used by parturient may be difficult to assess. Some press the demand button at the slightest discomfort, while others make only a few demands each hour. Patients are satisfied with the technique irrespective of their pattern of PCA use. PCEA group experienced the same degree of analgesia and satisfaction with comparable bupivacaine and fentanyl consumption. Patients in CIEA Group, however, used more bupivacaine and fentanyl than Groups PCEA alone or in combination. This finding is in keeping with other reports given by Gambling et al, ${ }^{3}$ which have shown that PCEA is associated with a dose-sparing effect. Our results have shown that PCEA is as effective as CIEA with less bupivacaine and less fentanyl consumption in the PCEA groups combined.

Patient-controlled epidural analgesia provides a means of comparing one local anaesthetic with another in various concentrations with or without different opioid supplements. In a study by Gambling et al, 3 the addition of fentanyl $2.5 \mu / \mathrm{mL}$ to bupivacaine $0.125 \%$ with $1: 400,000$ epinephrine resulted in measurable levels of fentanyl in the plasma of some samples. No patient suffered sequelae from fentanyl apart from mild pruritus. All fentanyl concentrations were usually associated with a plasma fentanyl concentration $>1.0 \mathrm{ng} / \mathrm{mL}$ volume. Patients in each group experienced similar degrees of satisfactory pain relief. All PCEA groups had lower bupivacaine and fentanyl consumption. No parturient or newborn had a clinically significant serum fentanyl concentration. PCEA using bupivacaine $0.125 \%$ and fentanyl $2.5 \mathrm{mcg} / \mathrm{mL}$ is a safe and effective technique regardless of which initial dose and lockout interval is programmed. We could not measure the serum fentanyl and bupivacaine levels, which is one of the drawbacks of this study.

Boselli et al ${ }^{12}$ studied in 133 parturients the use of PCEA alone or PCEA with basal infusion in labour analgesia and found that verbal pain scores, number of supplemental boluses and maternal satisfaction were same. Lim et al ${ }^{13}$ studied 300 nulliparous parturients with demand-only PCEA and PCEA with background infusion, the demand-only PCEA with a 5-mL bolus, 15-mins lockout interval resulted in less local anaesthetic consumption, but an increased incidence of breakthrough pain, higher pain scores, shorter duration of effective analgesia and lower maternal satisfaction when compared with PCEA with background infusion.

Ocampo et al $^{14}$ studied the use of PCEA alone to PCEA with 5 or $10 \mathrm{~mL} /$ hour of continuous basal infusion of $0.125 \%$ bupivacaine and $2 \mathrm{mcg} / \mathrm{mL}$ of fentanyl in labour analgesia and found that there was a reduced incidence of breakthrough pain and maximum pain scores in patients receiving PCEA with basal infusion. Maternal satisfaction was also higher in these patients.

Our study showed that demand-only PCEA without background infusion resulted in less local anaesthetic consumption, the incidence of breakthrough pain was very minimal and not statistically significant $p<0.0675$. This finding is in keeping with other reports, which have shown that PCEA is associated with a dose-sparing effect compared to continuous epidural infusion. $3,7,15$ This finding is even more pronounced when there is no background or basal infusion used with PCEA. Our results have shown that bolus-only PCEA is as effective as CIEA with $45 \%$ less bupivacaine and $36 \%$ less fentanyl consumption in the PCEA groups. The redundancy of a background infusion with PCEA has also been shown in studies by Paech, ${ }^{7}$ Ferrante and Lu L $\mathrm{L}^{15}$ et al demonstrated a $47 \%$ dose-sparing of bupivacaine and a $55 \%$ sparing of fentanyl when demand-dosing PCEA was compared to CIEA. Paech showed a dose-sparing of fentanyl, but not bupivacaine, in those patients receiving PCEA without a basal infusion. Degree of analgesia, maternal satisfaction and side effects were similarly unaffected by the addition of a basal infusion. Similar conclusions were made in another study, which compared bolus-only PCEA with.

In our study motor weakness was more in CIEA group. This is more significant in labour analgesia as high motor weakness can lead to unanticipated instrumental delivery as per the literature, even though it is controversial. ${ }^{16}$ In PCEA group, 34 parturients delivered spontaneously (SVD) compared to 23 in CIEA group. This has not been observed in other studies. There are many factors, which can affect SVD rate, but cannot be controlled. This observation does not imply a cause and effect relationship between PCEA and lower rates of SVD. A randomised controlled study with larger numbers would be needed to confirm this finding.

In our study on evaluation of the satisfaction index of the use of labour analgesia in labour, in the CIEA group 70\% preferred to use it next time as compared to the PCEA group, wherein $95 \%$ preferred to use it next time. This was statistically very highly significant with a $\mathrm{p}$ value of $<0.001$. High patient satisfaction was reported by most patients in this study either by VAS scores or by the global evaluation completed at the end of the study. Previous studies by Gambling et $\mathrm{al}^{3}{ }^{3}$ Tan et al, ${ }^{9}$ Curry et al, ${ }^{10}$ Sia $\mathrm{AT}^{11}$ have also demonstrated high patient acceptance and satisfaction, making this PCEA technique an increasingly popular method of labour analgesia.

In summary, patients in PCEA group experienced higher degree of satisfactory pain relief than IB group. No parturient or newborn had a clinically important side effects. PCEA using 
bupivacaine $0.125 \%$ with fentanyl $2.5 \mathrm{mcg} / \mathrm{mL}$ is a safe and effective technique. Reasonable hourly maximum doses should not be exceeded and minimum effective hourly doses should be guaranteed.

\section{REFERENCES}

1. Van der Vyver M, Halpern S, Joseph G. Patient-controlled epidural analgesia versus continuous infusion for labour analgesia: a meta-analysis. Br J Anaesth 2002;89(3):45965.

2. Taylor HJ. Clinical experience with continuous epidural infusion of bupivacaine at $6 \mathrm{ml}$ per hour in obstetrics. Can Anaesth Soc J 1983;30(3 pt 1):277-85.

3. Gambling DR, Cole PYC, McMorland GH, et al. A comparative study of patient controlled epidural analgesia and continuous infusion epidural analgesia during labour. Canadian Journal of Anaesthesia 1988;35(3):249-54.

4. Gambling DR, Huber CJ, Berkowitz J, et al. Epidural analgesia in labour: varying bolus dose and lockout interval. Can J Anaesth 1993;40(3):211-7.

5. Ross SM. Introduction to probability and statistics for engineers and scientists. $4^{\text {th }}$ edn. Villey publication, Elsevier, Newyork, California, Burkley 1987:pgs 106.

6. Moir DD. Extradural analgesia for caesarean section. Br J Anaesth 1979;51(2):79-80.

7. Paech MJ. Epidural analgesia in labour: constant infusion plus patient-controlled boluses. Anaesth Intensive Care 1991;19(1):32-9.

8. Boutros A, Blary S, Bronchard R, et al. Comparison of intermittent epidural bolus, continuous epidural infusion and patient controlled-epidural analgesia during labour. International Journal of Obstetric Anaesthesia 1999;8(4):236-41.
9. Tan S, Reid J, Thorburn J. Extradural analgesia in labour: complications of three techniques of administration. Br J Anaesth 1994;73(5):619-23.

10. Curry PD, Pacsoo C, Heap DG. Patient-controlled epidural analgesia in obstetric anaesthetic practice. Pain 1994;57(1):125-7.

11. Sia AT, Chong JL. Epidural $0.2 \%$ ropivacaine for labour analgesia: parturient-controlled or continuous infusion? Anaesth Intensive Care 1999;27:154-8.

12. Boselli E, Debon R, Cimino Y, et al. Background infusion is not beneficial during labor patient-controlled analgesia with $0.1 \%$ ropivacaine plus $0.5 \mathrm{~g} / \mathrm{ml}$ sufentanil. Anaesthesiology 2004;100(4):968-72.

13. Lim Y, Sia AT, Ocampo CE. Comparison of computer integrated patient controlled epidural analgesia vs. conventional patient controlled epidural analgesia for pain relief in labour. Anaesthesia 2006;61(4):339-44.

14. Lim Y, Ocampo CE, Supandji M, et al. A randomised controlled trial of three PCEA regimens for labour. Anaesth Analg 2008;107(6):1968-72.

15. Ferrante FM, Lu L, Jamison SB, et al. Patient-controlled epidural analgesia: demand dosing. Anaesth Analg 1991;73(5):547-52.

16. Smedstad KG, Morrison DH. A comparative study of continuous epidural infusion and intermittent epidural infusion for labour and analgesia. Canadian Journal of Anaesthesia 1988;35(3):234-41. 